\title{
On The Homogeneous Biquadratic Equation With 5 Unknowns $x^{4}-y^{4}=26\left(z^{2}-w^{2}\right) R^{2}$
}

\author{
Dr. P. Jayakumar ${ }^{1}$, J. Meena ${ }^{2}$ \\ ${ }^{1}$ Professor of Mathematics, Periyar Maniammai University, Vallam, Thanajvur-613 403, Tamil Nadu, India
}

${ }^{2}$ Ph.D. Scholar, Assistant Professor of Mathematics, A.V.V.M. Sri Pushpam College (Autonomous), Poondi -613 503, Thanajvur. ,Tamil Nadu, India

\begin{abstract}
The Homogenous biquadratic equation with five unknowns given by $x^{4}-y^{4}=26\left(z^{2}-w^{2}\right) R^{2}$ is considered and analyzed for finding its non zero distinct integral solutions. Introducing the linear transformations $x=u+v, y=u-v, z=2 u v+1, w$ $=2 u v-1$ and employing the method of factorization different patterns of non zero distinct integer solutions of the equation under the above equation are obtained. A few interesting relations between the integral solutions and the special numbers namely Polygonal numbers, Star number, Pronic number and Gnomonic number are exhibited.
\end{abstract}

Keywords: Homogeneous equation, Integral solutions, Polygonal number and special number

2010 Mathematics Subject Classification: 11D09

Notations used:

$\mathrm{t}_{\mathrm{m}, \mathrm{n}}$ - Polygonal number of rank $\mathrm{n}$ with sides $\mathrm{m}$.

$\mathrm{s}_{\mathrm{n}}$ - Star number

$\mathrm{p}_{\mathrm{n}}$ - Pronic number

$\mathrm{G}_{\mathrm{n}}$ - Gnomonic number

\section{Introduction}

The theory of Diophantine equations offers a rich variety of fascinating problems. In particular biquadratic Diophantine equations, homogeneous and non-homogeneous have aroused the interest of numerous mathematicians since antiquity [1-12]. In this context one may refer [4-10] for various problems on the biquadratic Diophantine equations. However, often we come across non-homogeneous biquadratic equations and as such one may require its integral solution in its most general form. This paper concerns with the homogeneous biquadratic equation with five unknown $x^{4}-y^{4}=26\left(z^{2}-w^{2}\right) R^{2}$ for determining its infinitely many non-zero integral solutions. Also a few interesting properties among the solutions are presented.

\section{Method of Analysis}

The biquadratic equation with five unknowns to be solved for its non-zero distinct integral solution is

$$
x^{4}-y^{4}=26\left(z^{2}-w^{2}\right) R^{2}
$$

Consider the transformations

$$
x=\mathrm{u}+\mathrm{v}, \mathrm{y}=\mathrm{u}-\mathrm{v}, \mathrm{z}=2 \mathrm{uv}+1, \mathrm{w}=2 \mathrm{uv}-1
$$

On substituting (2) in (1), we get

$$
u^{2}+v^{2}=26 R^{2}
$$

\subsection{Pattern: I}

Assume $26=(5+\mathrm{i})(5-\mathrm{i})(4)$

and $R=a^{2}+b^{2}=(a+i b)(a-i b)(5)$
Using (4) and (5) in (3) and employing the method of factorization, we get.

$(\mathrm{u}+\mathrm{i} v)(\mathrm{u}-\mathrm{iv})=(5+\mathrm{i})(5-\mathrm{i})(\mathrm{a}+\mathrm{i} b)^{2}(\mathrm{a}-\mathrm{i} b)^{2}$ On equating the positive and negative factors, we get

$\mathrm{u}+\mathrm{iv}=(5+\mathrm{i})(\mathrm{a}+\mathrm{ib})^{2}$

$\mathrm{u}+\mathrm{i} v=(5-\mathrm{i})(\mathrm{a}-\mathrm{ib})^{2}$

On equating real and imaginary parts, we obtain

$\mathrm{u}=\mathrm{u}(\mathrm{a}, \mathrm{b})=5 \mathrm{a}^{2}-5 \mathrm{~b}^{2}-2 \mathrm{ab}$

$\mathrm{v}=\mathrm{v}(\mathrm{a}, \mathrm{b})=\mathrm{a}^{2}-\mathrm{b}^{2}+10 \mathrm{ab}$

On substituting $u$ and $v$ in (2) we get the values of $x, y, z$ and $\mathrm{w}$. The non-zero distinct integrals values of $\mathrm{x}, \mathrm{y}, \mathrm{z}, \mathrm{w}$ and $\mathrm{R}$ satisfying (1) are given by

$x=x(a, b)=6 a^{2}-6 b^{2}+8 a b$

$\mathrm{y}=\mathrm{y}(\mathrm{a}, \mathrm{b})=4 \mathrm{a}^{2}-4 \mathrm{~b}^{2}-12 \mathrm{ab}$

$\mathrm{z}=\mathrm{z}(\mathrm{a}, \mathrm{b})=2\left(5 \mathrm{a}^{4}+5 \mathrm{~b}^{4}-30 \mathrm{a}^{2} \mathrm{~b}^{2}+48 \mathrm{a}^{3} \mathrm{~b}-48 \mathrm{ab} \mathrm{b}^{3}\right)+1$

$\mathrm{w}=\mathrm{w}(\mathrm{a}, \mathrm{b})=2\left(5 \mathrm{a}^{4}+5 \mathrm{~b}^{4}-30 \mathrm{a}^{2} \mathrm{~b}^{2}+48 \mathrm{a}^{3} \mathrm{~b}-48 \mathrm{ab} \mathrm{b}^{3}\right)-1$

$\mathrm{R}=\mathrm{R}(\mathrm{a}, \mathrm{b})=\mathrm{a}^{2}+\mathrm{b}^{2}$

\section{Properties:-}

1. $x(\mathrm{a}, 1)+\mathrm{y}(\mathrm{a}, 1)-\mathrm{P}_{\mathrm{n}}-\mathrm{Sn}_{\mathrm{a}} \equiv 0(\operatorname{Mod} 11)$

2. $\mathrm{R}(1,2 \mathrm{a})-4 \mathrm{t}_{4, \mathrm{a}} \equiv 0(\operatorname{Mod} 1)$

3. $. z(2 \mathrm{a}, 1)-3[\mathrm{x}(\mathrm{a}, 1)+\mathrm{y}(\mathrm{a}, 1)]+26 \mathrm{t}_{4, \mathrm{a}}-\mathrm{G}_{6 \mathrm{a}} \equiv 0(\operatorname{Mod} 32)$

4. $x(a+1, a+1)-c_{16, a}+4 G_{a}=8$

5. y $(\mathrm{a}+1, \mathrm{a}+1)+\mathrm{t}_{26, \mathrm{a}}+\mathrm{t}_{30, \mathrm{a}}+14 \mathrm{t}_{4, \mathrm{a}} \equiv 0(\operatorname{Mod} 12)$

\subsection{Pattern: II}

Also 26 can be chosen in equation (3) as

$$
26=(1+\mathrm{i} 5)(1-5 \mathrm{i})
$$




\section{International Journal of Science and Research (IJSR) \\ ISSN (Online): 2319-7064}

Index Copernicus Value (2013): 6.14 | Impact Factor (2014): 5.611

Using (5) and (6) in equation (3) it is written in factorizable form as

$$
(\mathrm{u}+\mathrm{iv})(\mathrm{u}-\mathrm{iv})=(1+5 \mathrm{i})(1-5 \mathrm{i})(\mathrm{a}+\mathrm{ib})^{2}(\mathrm{a}-\mathrm{ib})^{2}
$$

On equating the positive and negative factors,

we get,

$(\mathrm{u}+\mathrm{iv})=(1+5 \mathrm{i})(\mathrm{a}+\mathrm{ib})^{2}$

$(\mathrm{u}-\mathrm{iv})=(1-5 \mathrm{i})(\mathrm{a}-\mathrm{ib})^{2}$

On equating real and imaginary parts, we have

$u=\mathrm{u}(\mathrm{a}, \mathrm{b})=\mathrm{a}^{2}-\mathrm{b}^{2}-10 \mathrm{ab}$

$v=\mathrm{v}(\mathrm{a}, \mathrm{b})=5 \mathrm{a}^{2}-5 \mathrm{~b}^{2}+2 \mathrm{ab}$

Substituting the values of $\mathrm{u}$ and $\mathrm{v}$ in (2), the non-zero distinct values of $\mathrm{x}, \mathrm{y}, \mathrm{z}, \mathrm{w}$ and $\mathrm{R}$ satisfying (1) are given by $x=x(a, b)=6 a^{2}-6 b^{2}-8 a b$

$\mathrm{y}=\mathrm{y}(\mathrm{a}, \mathrm{b})=-4 \mathrm{a}^{2}+4 \mathrm{~b}^{2}-12 \mathrm{ab}$

$\mathrm{z}=\mathrm{z}(\mathrm{a}, \mathrm{b})=2\left(5 \mathrm{a}^{4}+5 \mathrm{~b}^{4}-30 \mathrm{a}^{2} \mathrm{~b}^{2}+48 \mathrm{a}^{3} \mathrm{~b}-48 \mathrm{ab}^{3}\right)+1$

$\mathrm{w}=\mathrm{w}(\mathrm{a}, \mathrm{b})=2\left(5 \mathrm{a}^{4}+5 \mathrm{~b}^{4}-30 \mathrm{a}^{2} \mathrm{~b}^{2}+48 \mathrm{a}^{3} \mathrm{~b}-48 \mathrm{ab}^{3}\right)-1$

$\mathrm{R}=\mathrm{R}(\mathrm{a}, \mathrm{b})=\mathrm{a}^{2}+\mathrm{b}^{2}$

\section{Properties:-}

1. $x(\mathrm{a}, 1)+\mathrm{R}(\mathrm{a}, 1)-7 \mathrm{t}_{4, \mathrm{a}}-\mathrm{G}_{4 \mathrm{a}} \equiv 0(\operatorname{Mod} 4)$

2. $2 y(1, \mathrm{~b})-(\mathrm{R}(1,2 \mathrm{~b}))^{2}+\mathrm{G}_{12 \mathrm{~b}} \equiv 0$

3. $x(\mathrm{n}, \mathrm{n}-1)-\mathrm{G}_{6 \mathrm{n}}+\mathrm{S}_{\mathrm{n}}+2 \mathrm{t}_{4, \mathrm{n}}-\mathrm{G}_{\mathrm{n}}+3=0$

4. $y(2 a, 2 a)+48 t_{4, a}=0$

5. $R(a(a+1), 1)-P_{a}+t_{6, A}-2 t_{4, A}-2=0$

\subsection{Pattern: III}

Rewrite (3) as

$$
1 * \mathrm{u}^{2}=26 \mathrm{R}^{2}-v^{2}
$$

$$
\text { Assume } \mathrm{u}=12 \mathrm{a}^{2}-\mathrm{b}^{2}=(\sqrt{26} \mathrm{a}+\mathrm{b})(\sqrt{26} \mathrm{a}-\mathrm{b})
$$

$$
\text { Write } 1 \text { as } 1=(\sqrt{26}+5)(\sqrt{26}-5)
$$

Using (8) and (9) in (7) it is written in factorizable form as

$$
\begin{aligned}
(\sqrt{26}+5)(\sqrt{26}-5)(\sqrt{26} \mathrm{a}+\mathrm{b})^{2}(\sqrt{26} \mathrm{a}-\mathrm{b})^{2}=(\sqrt{26} \mathrm{R} \\
+\mathrm{v})(\sqrt{26} \mathrm{R}-\mathrm{v})
\end{aligned}
$$

On equating the rational and irrational parts, we get

$(\sqrt{26}+5)(\sqrt{26} a+b)^{2}=(\sqrt{26} R+v)$

$(\sqrt{26}-5)(\sqrt{26} a-b)^{2}=(\sqrt{26} R-v)$

On equating the real and imaginary parts, we get

$$
R=R(a, b)=26 a^{2}+b^{2}+10 a b
$$$$
\mathrm{v}=\mathrm{v}(\mathrm{a}, \mathrm{b})=130 \mathrm{a}^{2}+5 \mathrm{~b}^{2}+52 \mathrm{ab}
$$

Substituting the values of $\mathrm{u}$ and $\mathrm{v}$ in (2), the non - zero distinct integral values of $x, y, z, R$ and w satisfying (1) are given by

$x=x(a, b)=156 a^{2}+4 b^{2}+52 a b$

$\mathrm{y}=\mathrm{y}(\mathrm{a}, \mathrm{b})=104 \mathrm{a}^{2}-6 \mathrm{~b}^{2}-52 \mathrm{ab}$

$\mathrm{z}=\mathrm{z}(\mathrm{a}, \mathrm{b})=2\left(3380 \mathrm{a}^{4}-5 \mathrm{~b}^{4}+1352 \mathrm{a}^{3} \mathrm{~b}-52 \mathrm{ab}^{3}\right)+1$

$\mathrm{w}=\mathrm{w}(\mathrm{a}, \mathrm{b})=2\left(3380 \mathrm{a}^{4}-5 \mathrm{~b}^{4}+1352 \mathrm{a}^{3} \mathrm{~b}-52 \mathrm{ab}^{3}\right)-1$

$R=R(a, b)=26 a^{2}+b^{2}+10 a b$

\section{Properties:}

1. $R(n+1,1)-\mathrm{ct}_{16, \mathrm{n}}-18 \mathrm{t}_{4, \mathrm{n}}-\mathrm{G}_{27 \mathrm{n}} \equiv 0(\operatorname{Mod} 37)$

2. $x(n+1, n+2)+y(n+3,1)-c_{16, n}-308 t_{4, n}-G_{266 n} \equiv 0$ (Mod 208)
3. $R(1,2 n)-4 P n-8 G_{n} \equiv 0(\operatorname{Mod} 27)$

4. $x(2 n, 3 n)-1072 t_{4, n}=0$

5. $y(2 n, 3 n)-S_{n}-44 t_{4, n}-3 G_{n}=0$

\subsection{Pattern: 4}

Rewrite (3) as

$$
\begin{gathered}
1 * v^{2}=26 R^{2}-u^{2} \\
\text { Write } 1 \text { as } 1=\frac{(\sqrt{26}-1)(\sqrt{26}+1)}{25} \\
\text { Assume } v=26 a^{2}-b^{2}=(\sqrt{26} a+b)(\sqrt{26} a-b)
\end{gathered}
$$

Using (12) and (13) in (11), it is written in factorizable form as

$$
\begin{array}{r}
\frac{(\sqrt{26}-1)(\sqrt{26}+1)}{25}(\sqrt{26} a+b)^{2}(\sqrt{26} a-b) \\
=(\sqrt{26} R+u)(\sqrt{26} R-u)(14)
\end{array}
$$

On equating the rational and irrational factors, we get

$$
\left.\begin{array}{l}
\mathrm{R}=\mathrm{R}(\mathrm{a}, \mathrm{b})=\frac{1}{5}\left(26 \mathrm{a}^{2}+\mathrm{b}^{2}+2 \mathrm{ab}\right) \\
\left.\mathrm{u}=\mathrm{u}(\mathrm{a}, \mathrm{b})=\frac{1}{5}\left(26 \mathrm{a}^{2}+\mathrm{b}^{2}\right) 52 \mathrm{ab}\right)(15)
\end{array}\right\}
$$

Replacing a by $5 \mathrm{~A}$ and $\mathrm{b}$ by $5 \mathrm{~B}$ in the above equations (13) and (15), we get

$R=R(A, B)=130 A^{2}+5 B^{2}+10 A B$

$\mathrm{u}=\mathrm{u}(\mathrm{A}, \mathrm{B})=130 \mathrm{~A}^{2}+5 \mathrm{~B}^{2}+260 \mathrm{AB}$

$\mathrm{v}=\mathrm{v}(\mathrm{A}, \mathrm{B})=650 \mathrm{~A}^{2}-25 \mathrm{~B}^{2}$

On substituting the values of $u$ and $v$ in (2), the non -zero distinct integrals values of $\mathrm{x}, \mathrm{y}, \mathrm{z}, \mathrm{w}$ and $\mathrm{R}$ satisfying (1) are given by

$\mathrm{x}=\mathrm{x}(\mathrm{A}, \mathrm{B})=780 \mathrm{~A}^{2}-20 \mathrm{~B}^{2}+260 \mathrm{AB}$

$\mathrm{y}=\mathrm{y}(\mathrm{A}, \mathrm{B})=-520 \mathrm{~A}^{2}+30 \mathrm{~B}^{2}+260 \mathrm{AB}$

$\mathrm{z}=\mathrm{z}(\mathrm{A}, \mathrm{B})=2\left(84500 \mathrm{~A}^{4}-125 \mathrm{~B}^{4}+16900 \mathrm{~A}^{3} \mathrm{~B}-\right.$

$\left.6500 \mathrm{AB}^{3}\right)+1$

$\mathrm{w}=\mathrm{w}(\mathrm{A}, \mathrm{B})=2\left(84500 \mathrm{~A}^{4}-125 \mathrm{~B}^{4}+10900 \mathrm{~A}^{3} \mathrm{~B}-\right.$ $\left.6500 \mathrm{AB}^{3}\right)-1$

$R=R(A, B)=130 A^{2}+5 B^{2}+10 A B$

\section{Properties}

1. $R(A, 1)-x(A, 1)+650 t_{4, A}-135 G_{A} \equiv 0(\operatorname{Mod} 14)$

2. $R(1,2 n)-y(1,2 n)-20 P_{n}-t_{242, n}-t_{806, n}$

$+201 \mathrm{G}_{\mathrm{n}} \equiv 0(\operatorname{Mod} 389)$

3. $x(1,4 n)+t_{642, n}-t_{443, n}+361 G_{n} \equiv 0(\operatorname{Mod} 781)$

4. $y(1,3 B)+t_{4, B}-P_{B}-529 G_{B}-t_{542, B}+519=0$

5. $R(A+1, A)-t_{292, A}-207 G_{A} \equiv 0(\operatorname{Mod} 131)$

\subsection{Pattern: 5}

Write $(3)$ as $(\mathrm{u}+\mathrm{R})(\mathrm{u}-\mathrm{R})=(5 \mathrm{R}+\mathrm{v})(5 \mathrm{R}-\mathrm{v})(16)$

Which is expressed is the form of ratio as

$\frac{u+R}{5 R+v}=\frac{5 R-v}{u-R}=\frac{A}{B}, \mathrm{~B} \neq 0(17)$

This is equivalent to the following two equations,

$-\mathrm{u} A+\mathrm{R}(5 \mathrm{~B}+\mathrm{A})-\mathrm{VB}=0$

$\mathrm{u}+\mathrm{R}(\mathrm{B}-5 \mathrm{~A})-\mathrm{VA}=0$

On solving the above equations by the method of cross multiplication we get,

$\mathrm{u}=\mathrm{u}(\mathrm{A}, \mathrm{B})=-\mathrm{A}^{2}-\mathrm{B}^{2}$

$\mathrm{R}=\mathrm{R}(\mathrm{A}, \mathrm{B})=\mathrm{A}^{2}+\mathrm{B}^{2}$

$v=v(A, B)=-5 A^{2}+5 B^{2}+2 A B$ 


\section{International Journal of Science and Research (IJSR) \\ ISSN (Online): 2319-7064}

Index Copernicus Value (2013): 6.14 | Impact Factor (2014): 5.611

Substituting the values of $u$ and $v$ in (2), the non - zero distinct integral values of $x, y, z, w$ and $R$ satisfying (1) are given by,

$\mathrm{x}=\mathrm{x}(\mathrm{A}, \mathrm{B})=-6 \mathrm{~A}^{2}+4 \mathrm{~B}^{2}+2 \mathrm{AB}$

$\mathrm{y}=\mathrm{y}(\mathrm{A}, \mathrm{B})=4 \mathrm{~A}^{2}-6 \mathrm{~B}^{2}-2 \mathrm{AB}$

$\mathrm{z}=\mathrm{z}(\mathrm{A}, \mathrm{B})=2\left[5 \mathrm{~A}^{4}-5 \mathrm{~B}^{4}-2 \mathrm{~A}^{3} \mathrm{~B}-2 \mathrm{AB}^{3}\right]+1$

$\mathrm{w}=\mathrm{w}(\mathrm{A}, \mathrm{B})=2\left[5 \mathrm{~A}^{4}-5 \mathrm{~B}^{4}-2 \mathrm{~A}^{3} \mathrm{~B}-2 \mathrm{AB}^{3}\right]-1$

$\mathrm{R}=\mathrm{R}(\mathrm{A}, \mathrm{B})=\mathrm{A}^{2}+\mathrm{B}^{2}$

\section{Properties:}

1. $x(A, 1)+R(A, 1)+t_{8, A}+2 t_{4, A} \equiv 0(\operatorname{Mod} 5)$

2. $\mathrm{y}(\mathrm{n}, \mathrm{n}+1)-\mathrm{t}_{8, \mathrm{n}}+6 \mathrm{G}_{\mathrm{n}}+\mathrm{P}_{\mathrm{n}} \equiv 0(\operatorname{Mod} 2)$

3. $\mathrm{R}(2,2 \mathrm{n})-4 \mathrm{t}_{4, \mathrm{n}} \equiv 0(\operatorname{Mod} 4)$

4. $\mathrm{y}(2 \mathrm{~A}, \mathrm{~A})-\mathrm{S}_{\mathrm{n}}-3 \mathrm{G}_{\mathrm{A}}=0$

5. $\mathrm{R}(\mathrm{A}+1, \mathrm{~A}-1)-2 \mathrm{t}_{4, \mathrm{~A}} \equiv 0(\operatorname{Mod} 2)$

\section{Conclusion}

It is worth to note that in (2), the transformations for $\mathrm{z}$ and $\mathrm{w}$ may be considered as $\mathrm{z}=2 \mathrm{u}+\mathrm{v}$ and $\mathrm{w}=2 \mathrm{u}-\mathrm{v}$. For this case, the values of $x, y$ and $R$ are the same as above where as the values of $\mathrm{z}$ and $\mathrm{w}$ changes for every pattern. To conclude one may consider biquadratic equations with multivariables $(\geq 5)$ and search for their non-zero distinct integer solutions along with their corresponding properties.

\section{References}

[1] Dickson, L.E., History of theory of numbers, Vol.11, Chelsea publishing company, New -York (1952).

[2] Mordell, L.J., Diophantine equation, Academic press, London (1969) Journal of Science and Research, Vol (3) Issue 12, 20-22 (December-14)

[3] Jayakumar. P, Sangeetha, K "Lattice points on the cone $x^{2}+9 y^{2}=50 z^{2}$ " International Journal of Science and Research, Vol (3), Issue 12, 20-22 (December -2014)

[4] Jayakumar P, Kanaga Dhurga, C," On Quadratic Diopphantine equation $\mathrm{x}^{2}+16 \mathrm{y}^{2}=20 \mathrm{z}$ " Galois $\mathrm{J}$. Maths, 1(1) (2014), 17-23.

[5] Jayakumar. P, Kanaga Dhurga. C, "Lattice points on the cone $x^{2}+9 y^{2}=50 z^{2}$ " Diophantus J. Math, 3(2) (2014), $61-71$

[6] Jayakumar. P, Prabha. S "On Ternary Quadratic Diophantine equation $\mathrm{x}^{2}+15 \mathrm{y}^{2}=14 \mathrm{z}^{2}$ " Archimedes J. Math., 4(3) (2014), 159-164.

[7] Jayakumar, P, Meena, J "Integral solutions of the Ternary Quadratic Diophantine equation : $x^{2}+7 y^{2}=$ $16 z^{2}$ " International Journal of Science and Technology, Vol.4, Issue 4, 1-4, Dec 2014.

[8] Jayakumar. P, Shankarakalidoss, G "Lattice points on Homogenous cone $\mathrm{x}^{2}+9 \mathrm{y}^{2}=50 \mathrm{z}^{2}$ " International journal of Science and Research, Vol (4), Issue 1, 2053-2055, January -2015 .

[9] Jayakumar. P, Shankarakalidoss. G "Integral points on the Homogenous cone $x^{2}+y^{2}=10 z^{2}$ " International Journal for Scienctific Research and Development, Vol (2), Issue 11, 234-235, January -2015

[10] Jayakumar.P, Prabha.S "Integral points on the cone $x^{2}$ $+25 \mathrm{y}^{2}=17 \mathrm{z}^{2}$ " International Journal of Science and Research Vol(4), Issue 1, 2050-2052, January-2015.
[11] Jayakumar.P, Prabha. S, "Lattice points on the cone $\mathrm{x} 2$ $+9 \mathrm{y} 2=26 \mathrm{z} 2$ "International Journal of Science and Research Vol (4), Issue 1, 2050-2052, January -2015

[12] Jayakumar. P, Sangeetha. K, "Integral solution of the Homogeneous Biquadratic Diophantine equation with six unknowns: $\left(\mathrm{x}^{3}-\mathrm{y}^{3}\right) \mathrm{z}=\left(\mathrm{W}^{2}-\mathrm{P}^{2}\right) \mathrm{R}^{4}$ "International Journal of Science and Research, Vol(3), Issue 12, 1021-1023 (December-201

\section{Author Profile}

P. Jayakumar received the B. Sc, M.Sc degrees in Mathematics from Madras University in 1980 and 1982 and the M. Phil, Ph.D degrees in Mathematics from Bharathidasan University , Thiruchirappalli in 1988 and 2010. Who is now working as Professor of Mathematics, Periyar Maniammai University,Vallam, Thanajvur-613403,

Tamil Nadu, India

J. Meena received the B. Sc, M.Sc degrees in Mathematics from Bharathidasan University, Thiruchirappalli in 2008 and in 2010 and the M. Phil., degree in Mathematics from Prist University, Thanjavur in 2013. Who is now working as Assistant Professor of Mathematics, A.V.V.M Sri Pushpam College Poondi (Autonomous), Thanjavur (District) - 613 503, Tamil Nadu, India. 